Ann. Biol. anim. Bioch. Biophys., r975, 15 (3), 547-558.

\title{
MESURE QUALITATIVE EN CONTINU DE L'ABSORPTION INTESTINALE DES GLUCIDES : EFFET DE L'INGESTION DE SACCHAROSE SUR LA CONCENTRATION EN GLUCOSE ET EN FRUCTOSE DANS LE SANG PORTE ET PÉRIPHÉRIQUE CHEZ LE PORC
}

\author{
A. AUMAITRE, A. RÉRAT, P. VAISSADE et P. VAUGELADE \\ avec la collaboration technique de A. Roger \\ Station de Recherches sur l'Elevage des Porcs, \\ Laboratoive de Physiologie de la Nutrition, \\ Centre national de Recherches zootechniques, I. N.R. A., \\ 78350 Jouy en Josas
}

RÉSUMÉ

On a mesuré en continu pendant 8 heures chez 3 porcs de 38 à $46 \mathrm{~kg}$ munis d'une canule de la veine porte et de la veine cave antérieure, la variation du taux sanguin de glucose vrai et de fructose, à la suite d'un repas à base de saccharose (400 ou $800 \mathrm{~g}$ ) additionné d'un mélange protidique minéral et vitaminique.

Les principaux résultats font apparaître deux types de réponse des animaux :

Dans un premier cas la glycémie préprandiale de l'animal est faible et l'absorption du glucose vrai entraîne une forte élévation persistante du taux de glucose dans la veine porte et ne provoque pas d'augmentation de la glucosémie périphérique, lors que le taux de fructose s'élève à la fois dans la veine porte (500 $\mathrm{mg} /$ litre) et dans le sang périphérique (200 $\mathrm{mg} /$ /itre).

Dans un second cas, chez un animal présentant une glycémie de jeûne élevée $(800 \mathrm{mg}$ de glucose/litre), l'ingestion du repas entraîne une augmentation parallèle du taux de glucose dans les deux veines, plus marquée toutefois dans le sang porte; la fructosémie augmente comme précédemment dans les deux veines atteignant au maximum $400 \mathrm{mg} /$ litre dans la veine porte.

On a, par ailleurs, estimé à partir des différences instantanées du taux des oses dans le sang, la vitesse d'absorption relative du fructose par rapport au glucose. Cette vitesse est de $60 \mathrm{p}$. Ioo de celle du glucose pour les animaux du premier type et de $74 \mathrm{p}$. Ioo dans le $2^{\mathrm{e}}$ cas. Les résultats apparaissent très reproductibles (quelle que soit la quantité de saccharose ingérée). 


\section{INTRODUCTION}

L'effet de l'ingestion d'un repas à base de glucides sur l'élévation de la glycémie du sang périphérique est bien connue chez les Monogastriques. Cepenđant, les méthodes classiques d'étude de la tolérance au glucose faisant appel par exemple à des prélèvements discontinus pendant un temps limité à I ou 2 heures, sont incapables de rendre compte du déroulement des processus de digestion et d'absorption intestinale, aussi bien chez l'Homme que chez le Porc (WiLson, I962). I) mêne, le seul dosage global des sucres réducteurs n'est valable que pour un glucide dont l'hydrolyse ne libère que $d u$ glucose (glucose, maltose, amidon et dérivés) alors qu'il est insuffisant dans le cas d'un diholoside complexe comme le saccharose. Nous avons ainsi préféré mesurer en continu, chez le Porc, la concentration en glucides du sang prélevé à la fois dans la veine porte (ARSAC et RÉRAT, I962) et dans la veine cave antérieure (RÉRAT et al., I973 et I974) afin d'exprimer qualitativement 1'absorption intestinale des glucides ingérés au cours d'un repas d'épreuve. De plus, la différence entre la vitesse d'absorption des différents oses est bien connue : ainsi, dès I 925 , CoRI démontrait chez le Rat, à l'aide d'anses intestinales isolées que le fructose était absorbé à une vitesse égale à la moitié de celle du glucose. Nous avons voulu mesurer spécifiquement chez le Porc l'évolution de la teneur sanguine en glucose vrai (Aumaitre et al., I969) ou en fructose (VAISSADE et al., I973), après un repas contenant le saccharose comme seule source de glucides.

\section{MATÉRIEL ET MÉTHODES}

\section{I. - Animaux}

Trois porcs mâles castrés pesant entre 38 et ${ }^{6} 6 \mathrm{~kg}$ sont rendus porteurs de deux doubles canules concentriques en polyéthylène souple introduites l'une dans la veine porte au niveau hépatique, l'autre dans la veine cave supérieure par la veine jugulaire externe. Les prélèvements de sang sont effectués en continu pendant 9 heures par jour à raison de $0,16 \mathrm{ml} / \mathrm{mn}$ sur chaque veine. Les animaux reçoivent par voie orale un anticoagulant dont on a vérifié l'inocuité : l'éthyldicoumarol à raison de $\mathbf{x}$ comprimé ( $150 \mathrm{mg}$ ) de biscoumacétate tous les deux jours, ce qui correspond à environ $4 \mathrm{mg}$ de matière active/kg de poids vif (LAPLACE, I972),

$$
\text { Conule "in vivo" }
$$

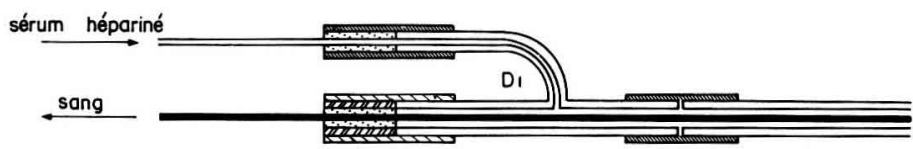

monchon normal WeA CPV violet CPV violet-noir CPV blanc

FIG. I. - Schéma de la canule de prélèvement en polvétlyylène

$$
\text { 2. - Dosages }
$$

Les dosages chimiques sur le sang sont effectués par dilution préalable au sérum physiologique à $9 \mathrm{p}$. I 000 de ClNa après une déprotéinisation par dialyse des glucides de faible poids 
moléculaire à travers une membrane de cellophane (membranne Cuprophane) qui présentel'avantage d'éviter la défécation du sang. Les mesures sont rendues quantitatives par le passage fréquent (toutes les heures) de solutions étalons de glucose et de fructose encadrant les taux sanguins mesurés; la dialyse s'opère contre une solution de sulfate de sodium pour le glucose et contre l'eau distillée pour le dosage du fructose (fig. $2 a$ et $2 b$ ). On dose ainsi les glucides présents principalement dans le plasma car on n'effectue pas d'hémolyse du sang avant la dialyse.

a

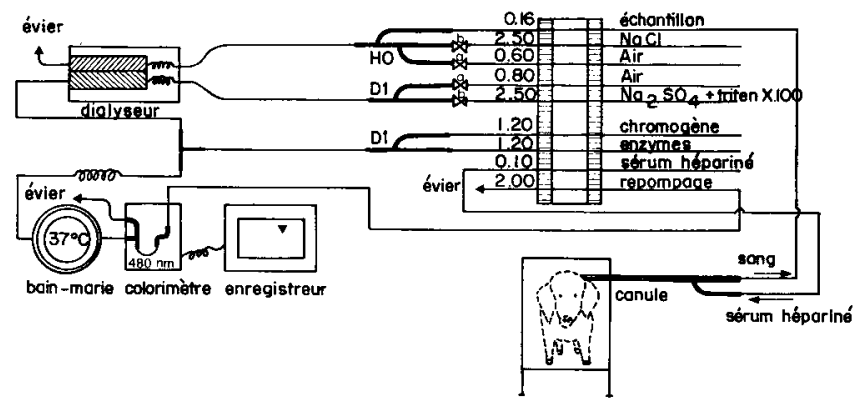

b

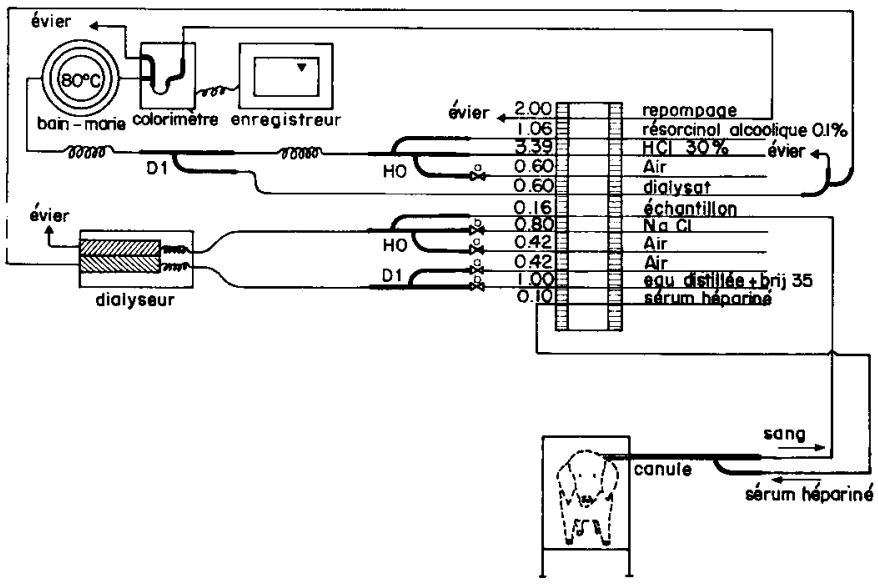

Fig. 2. - Dosage en continu des oses dans le sang

a) Dosage du glucose vrai

b) Dosage du fructose

Dosage spécifique du glucose par la glucose-oxydase.

Le sang est dilué par une solution saline à 9 p. I ooo avant la dialyse (fig. $2 a$ ); le dialysat est mis en présence des réactifs enzymatiques : la glucose-oxydase-péroxydase et un chromogène constitué par l'O-dianisidine.

Dans les conditions opératoires l'intensité de la coloration mesurée à $480 \mathrm{~nm}$ est proportionnelle à la dose de glucose (20o à 3 ooo $\mathrm{mg} / \mathrm{litre}$ ). Bien que les valeurs observées soient toujours en étroite corrélation, nous avons remarqué que les valeurs trouvées chez le Porc représentent 70 p. roo seulement des valeurs obtenues avec le dosage des sucres réducteurs lorsque l'on se réfère aux mêmes solutions témoins de glucose pur (VAISSADE et al., 1973).

\section{Fructose.}

Pour son dosage, nous avons adapté à 1'autoanalyseur, la méthode colorimétrique manuelle de ROE (I934) au résorcinol. Le liquide de contre dialyse du sang dilué est mis en présence d'une solution d'acide chlorhydrique à $30 \mathrm{p}$. 100 et de résorcinol alcoolique à $0, \mathrm{r}$ p. Ioo (fig. $2 \mathrm{~b}$ ). Le melange passe dans une bobine clouble et le passage dans un bain-maric (double bobine) à $80^{\circ} \mathrm{C}$ 
sont nécessaires. A cette température, on évite l'interférence due au glucose lorsque le taux de celui-ci est présent et ne dépasse pas $3 \mathrm{~g} /$ litre. La coloration est analysée au colorimètre avec un filtre interférentiel de $420 \mathrm{~mm}$ et comparée à un étalonnage effectué toutes les heures avec des solutions de fructose de titre connu. Il nous est ainsi possible de mesurer des concentrations en fructose de 25 à $750 \mathrm{mg} /$ litre. Malgré son manque relatif de spécificité la méthode de RoE fait apparaître un taux voisin de o dans le sang de l'animal à jeun; elle est sensible et reproductible.

Les mesures du taux de fructose et de glucose sont effectuées en continu ; cependant, toutes les ro minutes on alterne les deux dosages pour chaque veine, et on obtiendra des tracés discontinus des taux de glucose et de fructose, pouvant facilement être extrapolés entre des intervalles rapprochés.

\section{3. - Le repas d'épreuve}

Il comporte (tabl. I) soit $400 \mathrm{~g}$ ( 5 répétitions sur 3 porcs) soit $800 \mathrm{~g}$ (2 répétitions sur le même porc) de saccharose distribué le matin à 9 heures, après un jekne de $\mathrm{i} 8$ heures suivant un repas de même composition offert à volonté, favorable à une ingestion rapide et quantitative de la ration. Afin de placer l'animal dans une situation normale d'anabolisme, on ajoute au repas distribué sous forme de pâtée comprenant deux parties d'eau pour une partie d'aliment, un complément renfermant des protéines, des minéraux et des vitamines ${ }^{1}$ ) couvrant les besoins calculés d'un animal de même poids. Cette précaution est prise a fin de ne pas modifier l'équilibre de la composition du bol alimentaire du Porc, apportant notamment les minéraux (sodium) nécessaires à l'absorption active du glucose (RIKLIS et QUASTEL, I958).

TABLEAU I

\section{Repas d'épreuve effectués}

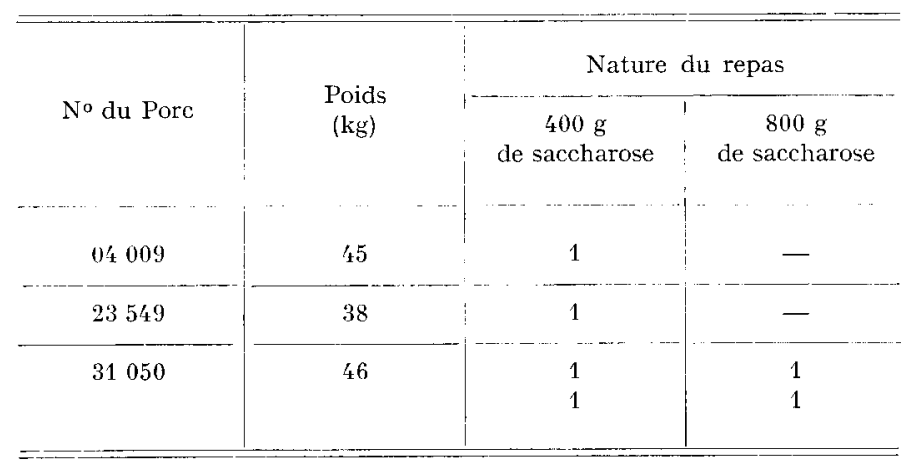

La présence de protéines renfermant les acides aminés indispensables est par ailleurs nécessaire en raison du risque d'augmentation anormale de la glycémie après ingestion du seul glucose, provoquée par l'absence de tyrosine (WoRBE et al., I968) qui entraîne une diminution de la perméabilité cellulaire au glucose chez le Rat.

Les résultats concernent l'évolution de la teneur en glucose et en fructose du sang porte et du sang cave antérieur I 5 minutes avant, pendant et 8 heures après l'ingestion du repas. Par ailleurs, on rapporte graphiquement comme le proposent DENCKER et al., I972, l'évolution de la différence instantanée des taux de glucose ou des taux de fructose entre les deux veines. Enfin, la différence entre l'intégrale des courbes de la glycémie porte et cave antérieure et de la fructosémie sur les 8 heures du test, permet d'apprécier en valeur relative la quantité de glucose et de fructose absorbés à la suite du repas. On peut en déduire une estimation de la vitesse relative d'absorption du fructose par rapport à celle du glucose chez le Porc. En fait, le pouvoir de captation du foie et la consommation d'oses par les tissus périphériques pour les deux oses ne sont pas

(1) La composition centésimale : tourteau de soja : 50; farine de poisson : I5; gluten de blé : I2; huile d'arachide : ro ; cellulose de bois : 4 ; complément minéral et vitaninique : 9 (I I p. Ioo de lipides ; 48 p. Ioo de protéines; I5 p. Ioo d'extractif non azoté). 
négligeables et varient avec la concentration sanguine, si bien que la différence de concentration mesurée concerne plutôt des différences de rendement de l'absorption du glucose et du fructose que des différences de vitesse d'absorption. L'importance relative des surfaces ainsi définies a été estimée par pesée de celles-ci après un tracé sur papier millimétrique à une échelle de $\mathrm{I} \mathrm{mm}$ par minute et de I $\mathrm{mm}$ par $5 \mathrm{mg}$ pour le taux de glucides.

\section{RÉSULTATS}

\section{I. - Evolution de la glycémie et de la fructosémie postprandiale}

Les principaux résultats sont présentés dans la figure 3 pour un repas de $400 \mathrm{~g}$ de saccharose. D'une façon schématique, on distingue pour une même quantité de glucide distribuée deux types de réponse du porc très caractéristiques et parfaitement reproductibles sur le même animal (tabl. 2) bien que le nombre d'essais soit faible, correspondant à 5 repas expérimentaux.

TABLEAU 2

Détermination de la vitesse relative d'absorption du fructose. par rapport au glucose selon l'animal, le type de réponse et la quantité de saccharose ingérée

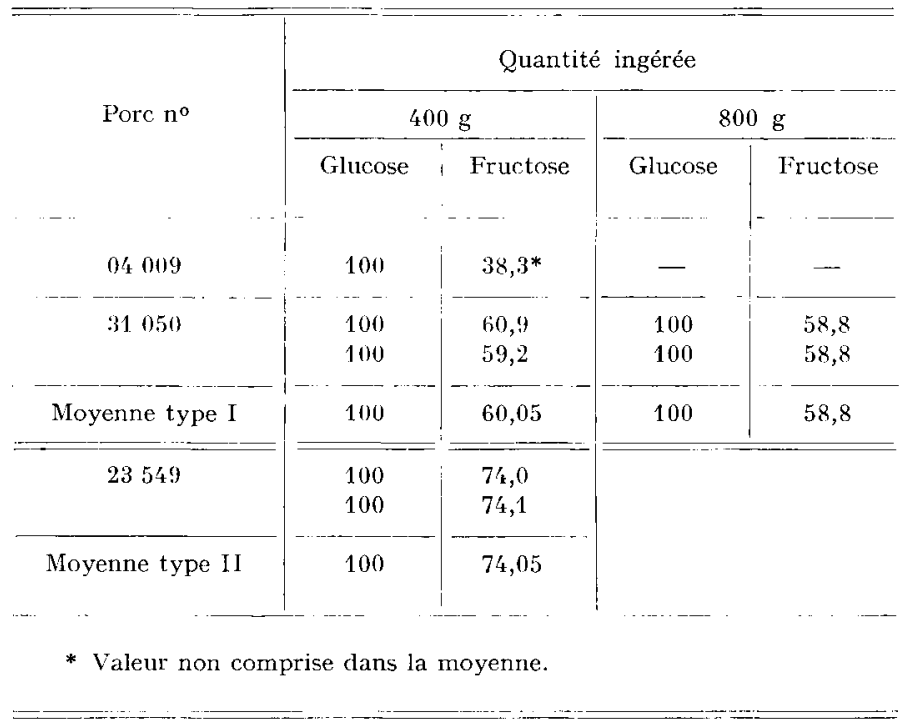

\section{a) Évolution du taux de glucose vrai.}

Dans le premier type de réponse (fig. $3 a$ ), on observe une augmentation du taux de glucose vrai de la veine porte à partir d'une valeur initiale faible et voisine de $550 \mathrm{mg} /$ litre dans les deux veines. Le maximum de la glycémie apparaît trente minutes après le repas, ensuite le taux de glucose présente de fortes variations. Cette augmentation est durable puisque la valeur initiale de la glycémie porte est à nouveau retrouvée entre $1 \mathrm{a} 7^{\mathrm{e}}$ et la $8^{\mathrm{e}}$ heure après le repas. Par contre, la glycémie de 
la veine cave après une légère augmentation immédiatement consécutive au repas, présente moins d'une heure après le début de celui-ci un retour à la valeur observée à jeun, voire même une valeur constante plus faible que la teneur initiale avant le repas.

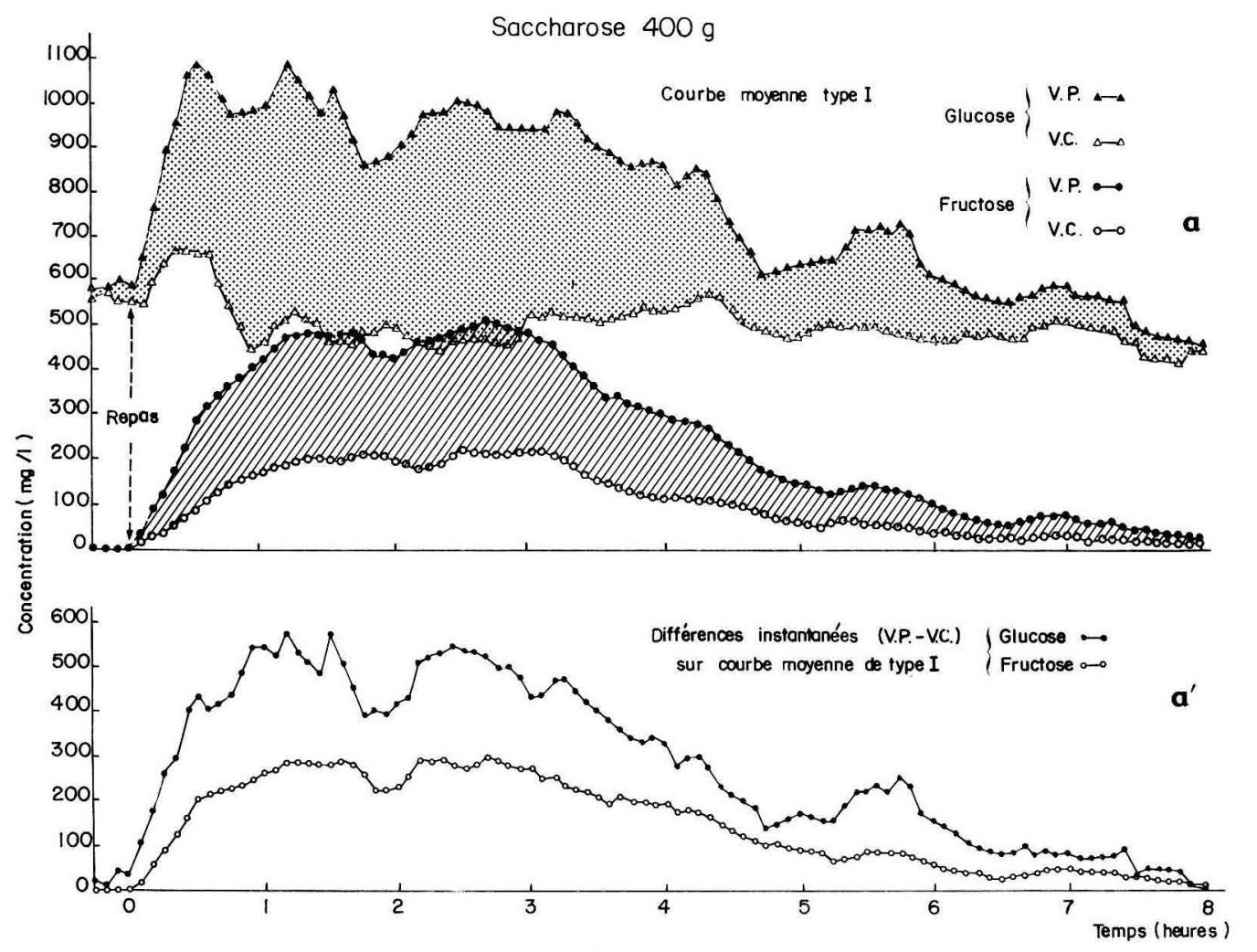

FIG. 3

$3 a$ : Évolution de la glycémie et de la fructosémie pré et postprandiale dans la veine porte et dans la veine cave antérieure.

$3 a^{\prime}$ : Difference entre le taux de glucose des deux veines et différence du taux de fructose.

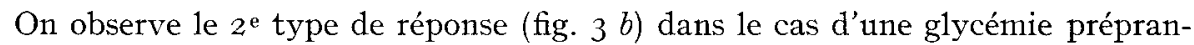
diale plus élevée (700 à $800 \mathrm{mg} / 1$ ) observée chez un autre animal après une durée de jeûne pourtant identique. L'évolution de la glycémie sanguine est similaire à celle décrite pour le premier type dans le seul cas de la glycémie porte. Par contre, on constate une augmentation de la glycémie de la veine cave qui augmente de plus de $300 \mathrm{mg} / \mathrm{l}$ par rapport à la valeur initiale observée à l'état de jeûne et qui se maintient pendant environ 6 heures après le repas. La glycémie périphérique suit sensiblement les variations de la glycémie porte et le phénomène se reproduit fidèlement chez le même animal à plusieurs jours d'intervalle. Les courbes correspondantes aux différences instantanées des taux de glucose dans les deux veines sont présentées à la figure $3 a^{\prime}$ et $3 b^{\prime}$. Elles présentent deux maxima assez caractéristiques dans le temps : le premier et le plus important est situé entre le début du repas et 2 heures, le second entre $2 \mathrm{~h} 30$ et $3 \mathrm{~h} 30$. 


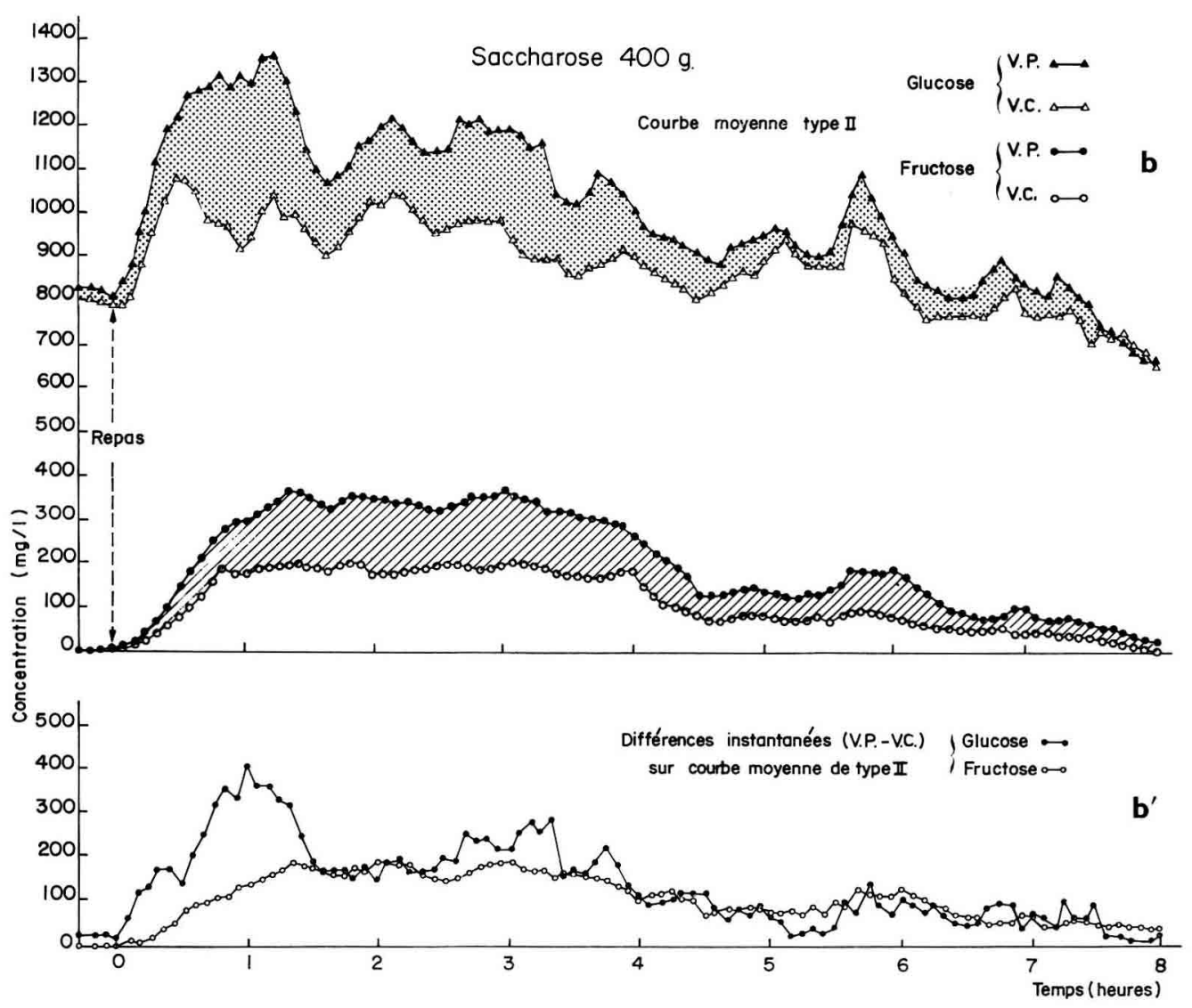

IIG. 3

$3 b$ : Glycémie et fructosémie pré et postprandiale (Animaux du type II).

$3 b^{\prime}$ : Différence des taux de glucose et différence des taux de fructose entre les deux veines.

b) Évolution de la fructosémie.

L'élévation du taux de fructose à la suite du repas à base de saccharose à partir d'une valeur inférieure à $\mathrm{I} O \mathrm{mg} /$ litre dans les deux veines (ou non mesurable) à jeun est beaucoup plus lente que dans le cas du glucose. Elle est cependant plus régulière dans le temps et ne présente pas de variations instantanées brusques et importantes. Toutefois, malgré de fortes atténuations, les pics de la glycémie correspondent à des variations perceptibles de la fructosémie. Dans tous les cas, la fructosémie porte maximum est obtenue environ une heure après le repas et se maintient à un niveau sensiblement constant pendant deux heures (jusqu'à trois heures après le repas) puis elle diminue lentement atteignant une valeur très faible $(25 \mathrm{mg} /$ litre $) 8$ heures après le début du repas.

Le niveau maximum de la fructosémie du sang porte varie cependant en valeur absolue suivant le type de réponse. Ainsi, dans le type I (fig. 3 a) la fructosémie maximum est voisine de $500 \mathrm{mg} /$ litre au moment du plateau ; elle n'est que de $35^{\circ} \mathrm{mg} / \mathrm{litre}$ dans le deuxième type (fig. 3 b). La fructosémie du sang périphérique varie parallè- 
lement à la fructosémie porte, présentant un plateau entre I et 3 heures après le repas. Toutefois, on observe peu de différence dans la valeur absolue des taux entre les deux types de courbes (fig. $3 a$ et $3 b$ ).

\section{c) Influence de la quantité de saccharose ingérée.}

Les résultats observés concernent deux répétitions du repas d'épreuve sur le même animal à deux jours d'intervalle.

Le profil général de l'évolution de la glycémie et de la fructosémie présenté à la figure 4 s'apparente au premier type observé (fig. $3 a$ ) pour des animaux présentant une faible glycémie avant le repas (550 $\mathrm{mg} /$ litre).

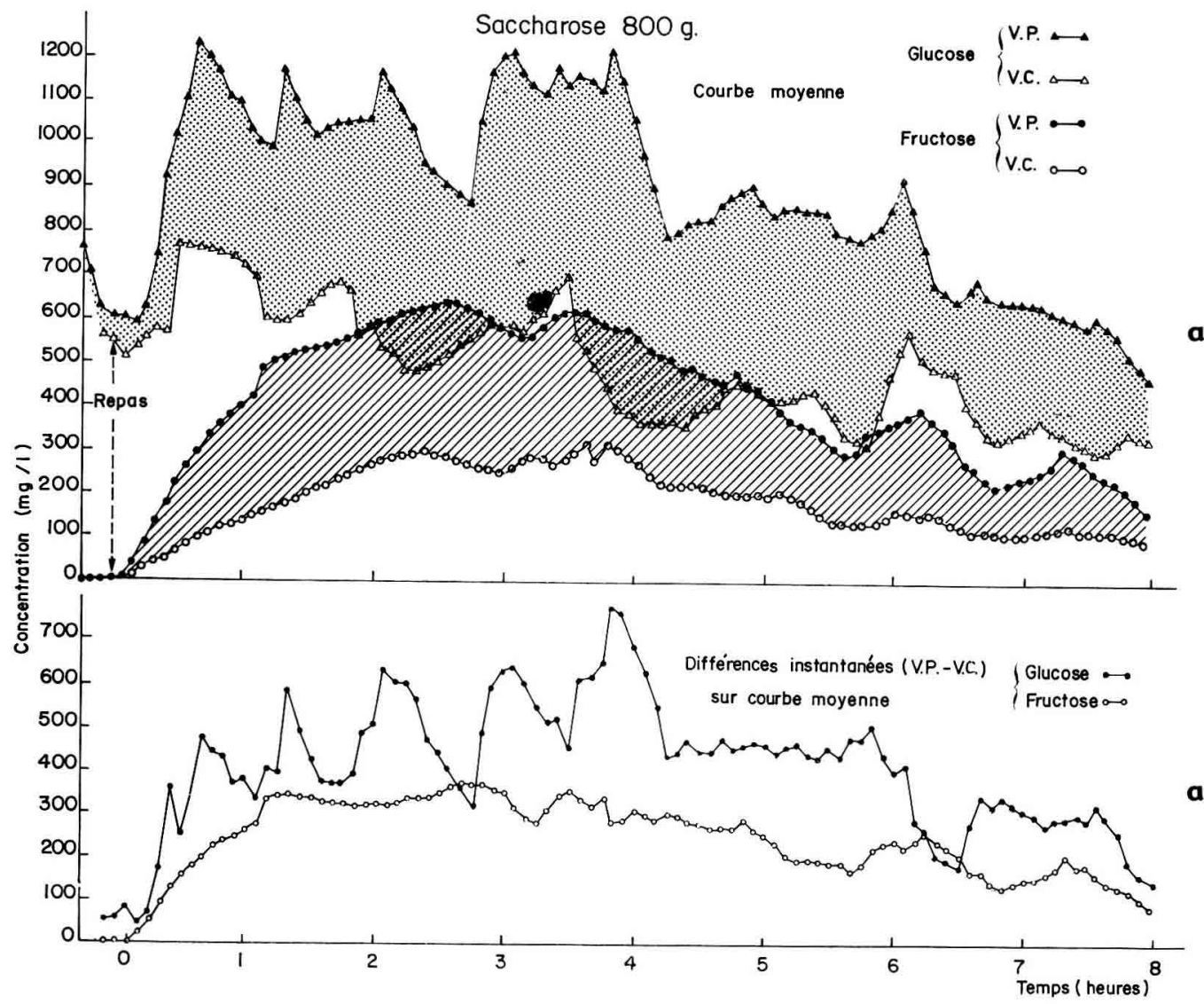

Fig. 4

$4 a$ : Glycémie et fructosémie pré et postprandiale (Animaux type I. $800 \mathrm{~g}$ de saccharose). $4 a^{\prime}$ : Différences des taux de glucose et des taux de fructose.

La glycémie vraie dans la veine porte présente de grandes variations instantanées la valeur maximum est atteinte 35 minutes après le début du repas; cette valeur élevée se maintient jusqu'à 4 heures après le repas et on retrouve approximativement la valeur initiale du jeûne vers 8 heures après le repas comme dans le cas précédent. 
La glycémie du sang de la veine cave, exceptée une légère augmentation suivant immédiatement le repas, retrouve deux heures après le repas la valeur initiale et s'abaisse irrégulièrement pendant toute la durée de l'observation. Enfin, 8 heures après le début du repas, on constate une différence notable entre le taux de glucose du sang porte et périphérique qui n'avait pas été observée avec un repas de $400 \mathrm{~g}$ et qui atteint $I_{5} 0 \mathrm{mg} /$ litre de sang.

La fructosémie porte présente une très forte élévation après le repas, avec toutefois un certain retard par rapport à l'augmentation du taux de glucose. Ainsi la valeur maximum est atteinte vers deux heures après le repas, présentant un plateau (voisin de $600 \mathrm{mg} /$ litre en valeur absolue) jusqu'à 4 heures. La fuctosémie du sang périphérique présente une évolution comparable à celle de la veine porte faisant apparaître notamment un plateau voisin de $25 \mathrm{mg}$ de fructose par litre entre 2 et 4 heures après le repas.

I'évolution des différences instantanées du taux de glucose ou de fructose des deux veines, présente une allure similaire au phénomène observé précédemment pour les animaux de type I. Cependant, pour le glucose, l'absorption (fig. 4 a) semble un phénomène très discontinu après un repas comportant une grande quantité de saccharose, le maximum de cette absorption semble se situer entre la $3^{\mathrm{e}}$ et la

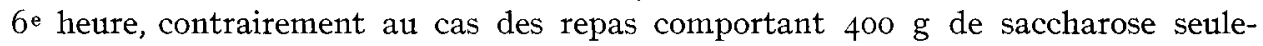
ment.

\section{2. - Estimation de la vitesse relative d'absorption du glucose et du fructose}

Après avoir estimé la surface correspondant aux différences instantanées du taux de glucose et de fructose des deux veines pour chaque repas d'épreuve, nous avons comparé la valeur relative (glucose $=$ Ioo) du rendement de l'absorption du fructose. Les valeurs observées (tabl. 2) font apparaître une différence suivant les types de courbes définies précédemment. Pour les animaux ne présentant pas d'augmentation de la glycémie périphérique vraie et mis à part le premier essai après un repas à base de saccharose (type I), on observe pour le fructose une valeur de 60 p. Ioo environ de celle du glucose, quelle que soit la quantité distribuée au cours $\mathrm{du}$ repas. Par contre, pour le deuxième type d'animal et bien qu'il ne soit pas possible de tester significativement les différences observées, cette valeur est notablement plus élevée (74 p. I00), sans qu'elle atteigne la même vitesse que le glucose. On peut souligner par ailleurs, malgré une importante variation de la valeur absolue des surfaces pour un même animal, une reproductibilité remarquable de la vitesse relative d'absorption du fructose par rapport au glucose.

\section{DISCUSSION}

Les principaux résultats relatifs à l'évolution du taux de glucose et de fructose du sang porte et cave font apparaître des données qualitatives intéressantes concernant la digestion du saccharose et l'absorption des deux oses libérés. Les animaux ne présentent aucune intolérance au saccharose même lorsque la quantité ingérée atteint $20 \mathrm{~g}$ par $\mathrm{kg}$ de poids vif et par repas; ces résultats sont en accord 
avec ceux de KIDDER et al. (I963), relatifs à la présence, à partir de 3 semaines d'âge, d'une quantité d'invertase intestinale suffisante chez le porcelet pour hydrolyser rapidement et quantitativement le saccharose. L'absorption du glucose apparait comme un phénomène discontinu dans le temps, et ces résultats confirment nos précédentes observations chez le Porc recevant d'autres types de glucides comme le glucose et l'amidon (AUMAITRE $c t$ al., I969 et I973) et sont retrouvés par ANDERSON (I974). L'absorption du fructose apparait par contre comme un phénomène beaucoup plus continu et beaucoup plus lent. Ceci semble être un phénomène général chez les monogastriques, notamment chez 1'Homme (DENCKER $t$ t al., I973). On doit cependant souligner chez le Porc une certaine variation de la glycémie du sang périphérique, déjà observée entre individus chez de nombreux animaux à la suite du repas, et probablement due à une différence considérable dans la tolérance aux glucides et notamment au glucose chez le Porc (Sownrs et al., r969). Toutefois, pour un même animal, on observe une très bonne répétabilité des tests, voire des constantes sanguines à l'état de jeûne.

Il apparaît deux types de réponses caractéristiques après l'ingestion de saccharose en ce qui concerne la variation de la seule glycémie vraie. Le premier type d'animal présente une hypoglucosémie du sang périphérique qui reste stable ou diminue dans le premier cas, ou au contraire augmente à la suite du repas et quelle que soit la quantité de saccharose ingérée. On peut alors faire deux hypothèses pour expliquer ce phénomène. Ainsi, les animaux du premier type peuvent être dans un état de jeûne intense et doivent reconstituer leurs réserves de glycogène hépatique, alors que chez les animaux du deuxième type la digestion $d u$ repas précédent se poursuit, maintenant un fort taux de glucose initial au moment du repas d'épreuve. La deuxième hypothèse conduirait à penser que les profils sanguins des porcs du premier type s'apparentent au cas pathologique d'intolérance au fructose entrainant une chute de la glucosémie du sang périphérique consécutif à la présence de fructose sanguin décrit chez 1'Homme (HËR, I957 ; MÉTAIs, I967; LANDAU et al., I97I).

Par ailleurs, nos résultats (fig. 3 et 4 ) font apparaitre une vitesse d'absorption plus faible du fructose par rapport au glucose. Cette notion est très classique depuis les essais de CORI (I925) sur des anses intestinales isolées chez le Rat; elle a été confirmée par de nombreux auteurs opérant in vitro (FRIDHANDLER et QUASTEL, I955) ou in vivo (Holdsworth et Dawson, I965) chez l'Homme. Cependant, notre estimation $\mathrm{du}$ rendement relatif d'absorption du glucose pourrait être entachée d'une erreur par défaut dans l'hypothèse d'une éventuelle conversion du fructose en glucose dans la paroi intestinale même, démontrée in vivo dans de nombreuses espèces (chien, cobaye, hamster et à un faible degré chez le Rat). Cependant, ce phénomène a été démenti chez 1'Homme anesthésié (Hor,Dsworth et Dawson, I965; DENKER et al., I972) et aucune donnée n'est disponible à notre connaissance chez le Porc. Il est difficile de retenir une telle hypothèse en raison de la très faible activité de la fructokinase intestinale (AHERNE et al., I 969). De même, la présence de fructose libre dans la lumière intestinale du Porc, en quantité supérieure au glucose (L/Y, I97I) permet de confirmer que les vitesses d'absorption intestinales sont différentes pour ces deux oses. Enfin, nos résultats ne tiennent pas compte d'une éventuelle transformation directe du fructose en lactate dans l'intestin ainsi que l'ont montré HoL,DsWORTH et IDAwSON (I 965 ) chez 1'Homme. On peut de plus affirmer surtout à l'examen des différences instantanées des taux sanguins entre les deux veines que l'absorption 
du glucose est très discontinue, alors que celle du fructose est plus continue dans le temps. Le fructose est métabolisé, puisqu'il disparaît du sang périphérique, ce qui est confirmé par la forte valeur en énergie métabolisable du saccharose chez le Porc et le faible taux retrouvé au niveau urinaire (AHERNE et al., rg69).

Malgré une légère imprécision du dosage du sang périphérique au niveau d'une veine plutôt que de l'artère, nous avons pu apprécier à l'aide d'une technique en continu, le rendement relatif de l'absorption des deux oses chez un animal éveillé et recevant un repas équilibré pendant un temps suffisamment long après l'ingestion. On peut souligner un certain nombre de particularités qualitatives concernant l'absorption discontinue du glucose, continue du fructose malgré la discontinuité de la vidange gastrique chez le Porc.

Reçu pour publication en mars 1975.

\section{SUMMARY}

CONTINUOUS QUALITATIVE MEASUREMENT

OF THE INTESTINAL, ABSORPTION OF CARBOHYDRATES :

EFFECT OF SUCROSE INGESTION ON THE PORTAI.

AND PERIPHERAL, BLOOD LEVEL OF GLUCOSE AND FRUCTOSE IN THE PIG

The variation in the blood levels of true glucose and of fructose after a meal containing sucrose $(400-800 \mathrm{~g})$ and a mixture of protein, minerals and vitamins was measured continuously for 8 hours in 3 pigs, between 38 and $46 \mathrm{~kg}$ live weight, fitted with portal vein and anterior caval vein cannulae.

On the basis of the main results, two types of responses were recorded in the animals :

In the first case, the preprandial blood level of glucose was low and the absorption of true glucose led to a large persistent rise of the glucose level in the portal vein; it did not bring about any increase in the peripheral blood level of glucose, whereas the level of fructose increased both in the portal vein (50o $\mathrm{mg} / \mathrm{litre}$ ) and in the peripheral blood, (200 $\mathrm{mg} / \mathrm{l}$ ).

In the second case, observed in an animal exhibiting a high blood level of glucose during fasting (80o $\mathrm{mg}$ glucose/litre), the intake of the meal led to a parallel increase in the glucose level of the two veins, however more marked in the portal vein. The blood level of fructose increased like previously in the two veins reaching to the maximum $400 \mathrm{mg} / \mathrm{litre}$ in the portal vein.

Furthermore, on the basis of instant differences in the blood level of monosaccharides, the relative absorption rate of fructose in comparison with glucose was estimated. This rate represented $60 \mathrm{p}$. Ioo as compared to that of glucose for the animals of the first type and 74 p. roo in the second case. Whatever the amount of sucrose ingested, the results are highly reproducible.

\section{RÉFÉRENCES BIBLIOGRAPHIQUES}

Aherne lí., Hays V. W., Ewan R. C., Speer V. C., I969. Absorption and utilization of sugars by the baby pig. J. Anim. Sci., 29, 444-450.

Anderson D. M., I974. The effect of feeding on the concentration of glucose and insulin in the portal and atrial plasma in pigs. J. Agvi. Sci. Camb., 82, 29-36.

Arsac M., Rérat A., I962. Technique de fistulation de la veine porte chez le Porc. Ann. Biol. anim. Bioch. Biophys., 2, 335-343.

Aumaitre A., Février C., Rérat A., Rigaud J., Thivend P., 1969. Application de l'analyse en continu à l'étude des variations de la glycémie du sang porte au cours de la digestion chez le Porc. C. R. Acad. Sci., Paris, 268, 717-720. 
Aumaitre A., Rérat A., Vaissade P., Valghlade P., i973. Étude expérimentale qualitative de l'absorption des glucides après ingestion d'un repas à base de glucose ou d'amidon. Ann. Biol. anim. Bioch. Biophys., 13, $784-788$.

Cori C. F., 1925. The fate of sugar in the aninal body. I. The rate of absorption of hexoses and pentoses from the intestinal tract. J. Biol. Chem., 66, 69I-715.

Dencker H., Lunderouist A., Meeuwisse G., Norryd C., Tranberg K. G., i972. Absorption of fructose as measured by portal catheterisation. Scand. J. Gastroenterology, 7, 7or-7o6.

Dencker H., Meevwisse G., Norryd C., Olin T., Tranberg K. G., 1973. Intestinal transport of carbohydrates as measured by portal catheterization in Man. Digestion, 9, 514-524.

Fridhander R., Quastel J. H., r955. Absorption of sugars from isolated swining intestine. Arch. Biochem. Biophys., 56, 412-423.

Hers H. G., I957. Le métabolisme du fructose. Arscia Ed., Bruxelles 250 p.

Holdsworth C. D., Dawson A. M., 1965. Absorption of fructose in Man. Proc. Soc. Exp. Biol. Med., 118, I $42-149$.

Kidder D. E., Manners M. J., McCrea M. R., I963. The digestion of sucrose by the piglet. Res. Vet. Sci., 4, I3I-I39.

LaPlace J. P., 1972. (Communication personnelle.)

Landat B. R., Marsinall J.S., Craig J. W., Hostetler K. Y., I97r, Quantitations of the pathways of fructose metabolism in normal and fructose intolerant subjects. J. Lab. Clin. Med., 76, 608-618.

Ly J., I971. Contribucion al estudio de la digestion de mieles en cerdos en crecimiento. Thesis, Universidad de La Habana, 48 pp.

Métais P., I967. Les dysglycidoses. Cah. Nutr. Diet., 2 (2), 23-31.

Rérat A., Février C., Aumaitre A., Vaissade P., Vaugelade P., I973. Absorption intestinale de quelques glucides chez le Porc. Cah. Nutr. Diét., 8, 154-156.

Rérat A., Aumaitre A., Vaissade P., Vaugelade P., I974. Mesure de l'absorption de glucides au cours de la digestion de l'amidon de mais chez le Porc. C. R. Acad. Sci., Paris, 279, Série D, $83 \mathrm{I}-834$.

Riklis E., QUASTEL J. H., 1958. Effect of cations on sugar absorption by isolated surviving guinea pig intestine. Canad. J. Biochem. Physiol., 36, 347-362.

Ros J.H., I934. Colorimetric method for the determination of fructose in blood and urine. J. Biol. Chem., 10\%, 15-22.

Sowers J. R., Burkeholder J. N., Tumbleson M. E., Guthrie R. A., ig69. Intravenous glucose tolerance test in Hormel Miniature swine. J. Comp. Lab. Med., 3, 47-5 I.

Vaissade P., Aumaitre A., Rérat A., ig73. Mesure en continu de la glycémie du sang porte et périphérique chez le Porc éveillé : application à l'étude de l'absorption intestiriale des glucides. Symp. Int. Pharmaceutique, 4-5 oct. I973, Paris, Technicon et Cie ed.

Wilson T. H., I962. Intestinal absorption. Saunders Co ed. Philadelphia., 263 p.

Worbe J. F., Darmenton P., Faure R., Guinand C., ig68. Contribution à l'étude des modifications du métabolisme glucidique consécutives à l'administration d'une dose unique de L-lysine et de L-tyrosine chez le Rat. J. Physiol. Paris, 60, 217-229. 